\title{
Total harmonic distortion of an asymmetric quasi-sinusoidal current
}

\author{
Vitaliy Grebennikov ${ }^{1, *}$, Irina Ermoeva $^{1}$, and Evgeniy Yaroslavtsev ${ }^{1}$ \\ ${ }^{1}$ Tomsk Polytechnic University, 634050 Tomsk, Russia
}

\begin{abstract}
This paper presents the correlation of factors that determine the quality of asymmetric quasi-sinusoidal output current and dynamic losses in the switches of the current generator circuit. The operating mode of the generator was obtained, especially of its power circuit elements, where combination of acceptable output current quality with relatively small dynamic losses in switches and mass-dimensional parameters of the inductor are provided. Achieved results can be used in designing this type of generators.
\end{abstract}

\section{Introduction}

Quasi-sinusoidal current is widely used in electrochemical and electrophysical technologies [1]. One of the generator circuits, which provides this type of current, is described in [2] and shown in Figure 1. The shape of half-waves of the generator output current which is shown in Figure 2 is quasi-sinusoidal because of its formation principle. This shape can be approximated to an ideal one by reducing the ripples of current and increasing the amount of switching cycles. It causes the increasing of power transistors switching frequencies and, as a result, the increasing of dynamic losses in switches of the current generator. Therefore, there is a problem how to choose an effective operating mode of generator power circuit elements where an acceptable output current quality combines with relatively small dynamic losses in switches and with small mass-dimensional parameters of the inductor.

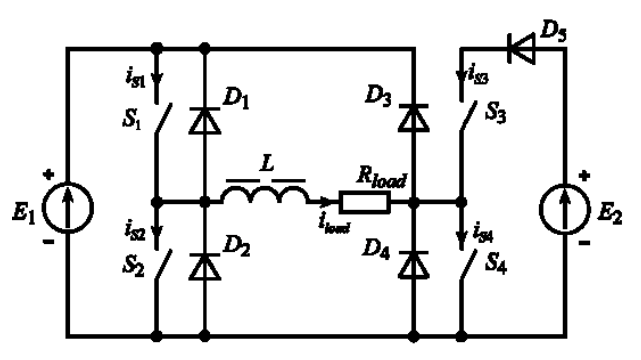

(a)

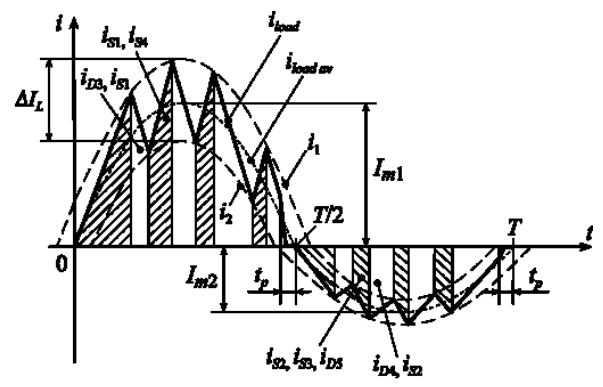

(b)

Fig. 1. Current generator circuit (a) and the current waveforms are explained of the circuit operation (b).

\footnotetext{
* Corresponding author: grebennikovvv@,tpu.ru
} 


\section{Materials and methods}

Total harmonic distortion (THD) is used to evaluate the output current quality of the generator. The value of this factor for asymmetric quasi-sinusoidal current depends on two parameters:

- non-ideality of each one of the half-waves (instead of an ideal sinusoidal shape) which are formed by half-waves current converter (as a result of formation principle);

- asymmetry of half-waves $S=I_{m 1} / I_{m 2}$.

Both of these parameters cause the appearance of higher harmonics, and it becomes the reason for harmonic factor to get definite values, which are different from zero. Let us examine a separate influence of the mentioned parameters on the THD of the asymmetric quasi-sinusoidal current, conducting the necessary research using OrCAD simulation. The definition of total harmonic distortion is set for first 40 harmonics in the THD calculation directive.

The circuit shown in Figure 1 was used for simulation. Parameter $S$ of the asymmetric quasi-sinusoidal current was equal $1(S=1)$ to exclude the influence of the asymmetry on the THD. The output current has a symmetric quasi-sinusoidal shape. To make modeling results objective, the THD of this quasi-sinusoidal current is equal to $12 \%$.

The following notations are used: $\Delta I_{L}$ - required value of current ripples; $K_{\text {rip }}=\Delta I_{L} / I_{m}-$ ripple factor of load and inductor current; $U^{*}=I_{m} \cdot R_{\text {load }} / E=U_{m \text { load }} / E$ - normalized value of the average amplitude of the load voltage with respect to the supply voltage (then - the normalized output voltage amplitude); $U_{m}$ load - the average amplitude of the voltage across the load; $\tau=L / R_{\text {load }}$ - the time constant; $\delta=T / \tau$ - the damping ratio of the transient showing how many times the period of a sine wave is higher than the time constant; $T-$ period of the output current; $f$ - frequency of the output current; $N$ - amount of switching cycles of switches in half-period of formed quasi-sinusoid.

\section{Results}

The results of the simulation are shown in Figure 2 and Figure 3. They are presented as characteristics of THD and $N$, and they depends on $\delta$ at various values of $K_{\text {rip }}$ and $U^{*}$.

Let us view these characteristics more in details. When $\delta$ is small THD has the biggest values on every graphs, and when $K_{\text {rip }}=0,3$ and $K_{\text {rip }}=0,4$ they are above permissible maximum limits $12 \%$. It is explained by respectively big ripples of the formed current and comparatively small number of switching cycles of switches $N$ that appear after long

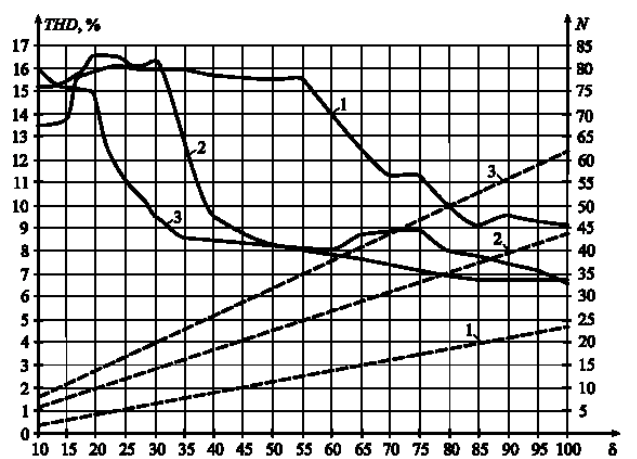

(e)

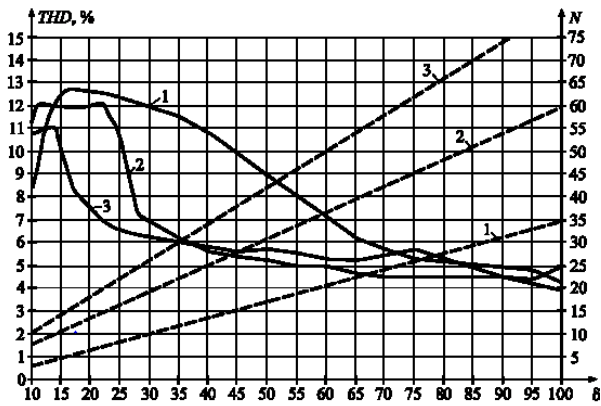

(b)

Fig. 2. THD (solid line) and $N$ (dashed line) vs the damping ratio $\delta$ at $K_{\text {rip }}=0,4$ (a) and $K_{\text {rip }}=0,3$ (b) for different $U^{*}: 1-U^{*}=0,8 ; 2-U^{*}=0,5 ; 3-U^{*}=0,2$. 


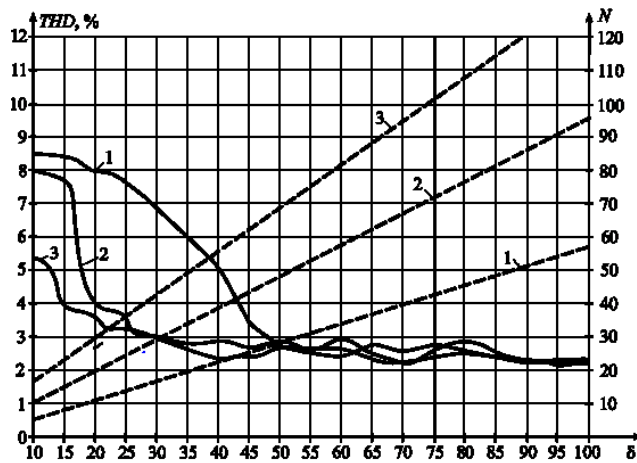

(a)

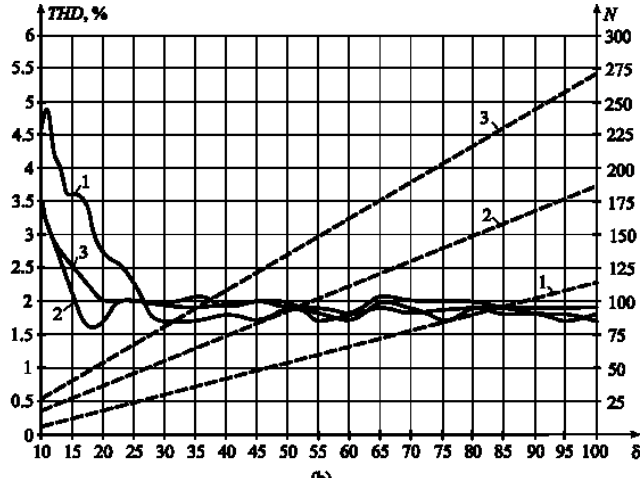

(b)

Fig. 3. THD (solid line) and $N$ (dashed line) vs the damping ratio $\delta$ at $K_{\text {rip }}=0,2$ (a) and $K_{\text {rip }}=0,1$ (b) for different $U^{*}: 1-U^{*}=0,8 ; 2-U^{*}=0,5 ; 3-U^{*}=0,2$.

transient in every cycle of the generator. Visible reduction of THD with growth of $N$ at the same time are observed from some minimal value of damping ratio $\delta_{\min }$. Moreover, as $K_{\text {rip }}$ becomes smaller, $\delta_{\text {min }}$ becomes smaller too. The speed of decreasing THD, when $\delta>\delta_{\text {min }}$, depends on ripple rate and normalized amplitude of the output voltage. For example, when $K_{\text {rip }}=0,3$ and $U^{*}=0,2\left(\delta_{\min } \approx 16\right)$, the speed of decreasing of THD is less than when $U^{*}=0,2\left(\delta_{\min } \approx 14\right)$ and $U^{*}=0,2\left(\delta_{\min } \approx 23\right)$. If damping ratio grows further, THD will come close to steady state value. This is seen especially when $K_{\text {rip }}=0,1$. It can be explained by the following, when some value of $N$ is reached, the farther increasing of the number of operating cycles in the half-period of the formed quasi-sinusoid wave does not lead to visible decreasing of THD. When $K_{\text {rip }}=0,4$, THD does not reach steady state values in the observed range. It should be noted that when $K_{\text {rip }}=0,1$ and $K_{\text {rip }}=0,2$, in the range of changing $\delta$ THD is not higher than $12 \%$. The mentioned characteristics allow to choose a near-optimal value of inductance for the current forming inductor which can provide relatively small dynamic losses in switches and the needed value of THD.

Obviously, the inductance of the current forming inductor should be as small as possible to reduce the calculated power of it. So, it is appropriate to make a formation of quasisinusoidal current when values of attenuation rate $\left(\delta=T / \tau=T \cdot R_{\text {load }} / L\right)$ are respectively high. In this case, low values of THD are provided. However, the number of operating cycles of switches $N$ with average frequency of transistors switching $\left(f_{S \text { av }}=2 \cdot N \cdot f\right)$ and level of dynamic losses can become unacceptably high. Therefore, the choice of the value of $\delta$ should be decided on the base of the complex parameters: power of current forming inductor, the value of THD, the average frequency of the transistors switching and the losses in them. Values of ripple factor recommended for practical using are commonly in range $K_{\text {rip }}=0,2 \div 0,3$.

Table 1. The results of decomposition in normalized (to $1^{\text {st }}$ harmonic) form.

\begin{tabular}{|c|c|c|c|c|c|c|c|c|c|c|}
\hline \multirow{2}{*}{ Current } & \multicolumn{10}{|c|}{ Number of harmonic } \\
\cline { 2 - 13 } & 1 & 2 & 3 & 4 & 5 & 6 & 7 & 8 & 9 & 10 \\
\hline $\mathrm{ASC}^{1} \times 10^{-3}$ & $10^{3}$ & 347 & 0 & 69 & 0 & 30 & 0 & 17 & 0 & 11 \\
\hline $\mathrm{AQC}^{1}, \times 10^{-3}$ & $10^{3}$ & 345 & 5.4 & 63 & 5.1 & 28 & 8.3 & 18 & 14 & 21 \\
\hline $\mathrm{SQC}^{1}, \times 10^{-3}$ & $10^{3}$ & 1.8 & 6.7 & 1.9 & 3.9 & 1.7 & 3.9 & 1.2 & 7.9 & 1.3 \\
\hline $\mathrm{AQC}^{2}, \times 10^{-3}$ & $10^{3}$ & 380 & 29 & 79 & 27 & 30 & 18 & 34 & 42 & 33 \\
\hline $\mathrm{SQC}^{2}, \times 10^{-3}$ & $10^{3}$ & 2.5 & 29.5 & 1.2 & 29.6 & 1.8 & 19.6 & 1 & 46 & 0.8 \\
\hline \multicolumn{10}{|c|}{$K_{\text {rip }}=0,2 ;{ }^{2} K_{\text {rip }}=0,3$} \\
\hline
\end{tabular}

To evaluate the influence of half-wave asymmetry on harmonic factor, spectra contents of asymmetrical sinusoidal current (ASC), asymmetrical quasi-sinusoidal current (AQC) 
and symmetrical quasi-sinusoidal current (SQC) were investigated.

Fourier series expansion of asymmetrical sinusoidal current on harmonics was made by known formulas, and analyzing of spectrums of AQC and SQC was made in PSpice with calculations of THD. The results of this decomposition in normalized (to $1^{\text {st }}$ harmonic) form for $K_{\text {rip }}=0,2$ and $K_{\text {rip }}=0,3$ when $S=10, \delta=20$, are shown in Table 1 and Table 2 .

Table 2. The results of decomposition in normalized (to $1^{\text {st }}$ harmonic) form.

\begin{tabular}{|c|c|c|c|c|c|c|c|c|c|c|}
\hline \multirow{2}{*}{ Current } & \multicolumn{10}{|c|}{ Number of harmonic } \\
\cline { 2 - 13 } & 11 & 12 & 13 & 14 & 15 & 16 & 17 & 18 & 19 & 20 \\
\hline $\mathrm{ASC}, \times 10^{-3}$ & 0 & 7.3 & 0 & 5.3 & 0 & 4.1 & 0 & 3.2 & 0 & 2.6 \\
\hline $\mathrm{AQC}^{1}, \times 10^{-3}$ & 13 & 6.8 & 19 & 25 & 17 & 12 & 26 & 28 & 21 & 9.9 \\
\hline $\mathrm{SQC}^{1}, \times 10^{-3}$ & 5 & 1.2 & 1.7 & 2.1 & 16 & 0.6 & 22 & 3 & 26 & 2.1 \\
\hline $\mathrm{AQC}^{2}, \times 10^{-3}$ & 25 & 21 & 19 & 29 & 12 & 44 & 69 & 33 & 13 & 32 \\
\hline $\mathrm{SQC}^{2}, \times 10^{-3}$ & 27 & 1 & 25 & 1.5 & 13.4 & 1.9 & 69 & 2 & 14 & 2.3 \\
\hline \multicolumn{10}{|c|}{$K_{\text {rip }}=0,2 ;{ }^{2} K_{\text {rip }}=0,3$} \\
\hline
\end{tabular}

Analyzing the data from tables, we can draw following results:

- The spectral composition of AQC is represented by even-numbered harmonics (the more significant are the $2^{\text {nd }}$, the $4^{\text {th }}$ and the $6^{\text {th }}$ ).

- The value of the asymmetric current THD is defined by the $2^{\text {nd }}$ harmonic, and its weight in the harmonic spectrum is in 5 and more times bigger than the other higher harmonics. The weight of the $2^{\text {nd }}$ harmonics of ASC and AQC is approximately the same, and their THD has not much difference (with $S=10$ and $K_{\text {rip }}=0,2$ THD of ASC is 35,61\%, and THD of AQC is 36,67\%).

- The weight of the low-order high harmonics in AQC spectrum is far more than the weight of the same harmonics of SQC spectrum. So, the half-wave asymmetry takes the main part in setting the value of THD.

- With increasing of the ripple factor there is also an increasing of the high harmonics weight in the spectrum of the formed SQC. It is explained by the increasing of THD. For example, when $K_{\text {rip }}=0,2$ the total harmonic distortion of SQC is $75 \%$ and when $K_{\text {rip }}=0,3$ it is $11,7 \%$.

\section{Conclusions}

The investigation of the asymmetric quasi-sinusoidal current harmonic composition and the symmetric quasi-sinusoidal current harmonic composition shows that the influence of halfwave asymmetry on the value of THD is much greater than the influence of non-ideality of symmetric quasi-sinusoidal current.

To provide THD of the symmetric quasi-sinusoidal current with value not above $12 \%$ and with the ripple factor equal to $(20 \div 30) \%$, the period of the formed current need to be bigger not less than in 10 times of magnitude of the time constant of the current forming circuit with the minimal load resistance.

\section{References}

1. B.A. Baginskiy, V.V. Grebennikov, S.V. Obraztsov, 6th Int. Scientific and Practical Conf. of Students, Postgraduates and Young Scientists "Modern Techniques and Technology”, 74 (2000)

2. B.A. Baginskii, V.V. Grebennikov, B.M. Nigof, D.N. Ogorodnikov, E.V. Yaroslavtsev, Instrum. Experim. Tech., 44(2), 243 (2001) 